\title{
Manifestaciones dermatológicas de la diabetes: clasificación y diagnóstico
}

\author{
Diana Patricia Crizón-Díaz' , Camilo Andrés Morales-Cardona²
}

\section{RESUMEN}

Se estima que en Colombia hay 2.836.500 adultos con diabetes, una enfermedad con una prevalencia del 8,4\%. La exposición a niveles elevados de glucosa afecta los procesos de proliferación y diferenciación en las células de todos los órganos y tejidos, así mismo en los queratinocitos, fibroblastos y demás células presentes en la piel, alteraciones que ocurren en más de un tercio de los diabéticos y que pueden ser la manifestación inicial de la enfermedad. La frecuencia y presentación de estas dermatosis varía según la población estudiađa. La dermopatía diabética, la acantosis nioricans, los fibromas laxos, el prurito y la xerosis son las formas más comunes y se consideran marcadores cutáneos de la diabetes. La presente revisión se enfoca en las manifestaciones dermatológicas específicas y no específicas de la diabetes, así como en las relacionadas con su tratamiento. Estas pueden ser un reflejo del estado metabólico actual o previo del paciente diabético y su oportuna identificación permite orientar el diagnóstico primario, sospechar un estado prediabético u optimizar el tratamiento de la enfermedad en los individuos ya diagnosticados.

\section{PALABRAS CLAVE}

Dermatología; Diabetes Mellitus; Manifestaciones Cutáneas

\section{SUMMARY}

\section{Diabetes mellitus and the skin}

It is estimated that in Colombia there are 2,836,500 adults with diabetes, and that the disease has a prevalence of $8.4 \%$. Exposure to elevated glucose levels affects the processes of cell

1 Médica Dermatóloga. Hospital Universitario Centro Dermatológico Federico Lleras Acosta E.S.E. Bogotá, D.C., Colombia.

2 Médico Dermatólogo. Hospital Universitario Centro Dermatológico Federico Lleras Acosta E.S.E. Bogotá, D.C., Colombia. Docente Adjunto. Fundación Universitaria Sanitas. Bogotá, D.C., Colombia.

Correspondencia: Diana Patricia Crizón-Díaz; dianac4d@gmail.com

Recibido: julio 4 de 2019

Aceptado: diciembre 11 de 2019

Cómo citar: Crizón-Díaz DP, Morales-Cardona CA. Manifestaciones dermatológicas de la diabetes: clasificación y diagnóstico. latreia. 2020 Jul-Sep;33(3):239-50. D0I 10.17533/ udea.iatreia.58. 
proliferation and cell differentiation in all organs and tissues, as well as keratinocytes, fibroblasts, melanocytes and other cells present in the skin, alterations that occur in more than one third of diabetics and which may be the initial manifestation of the disease. The frequency and presentation of these dermatoses varies according to the population studied, with a predominance of fungal and bacterial infections. Diabetic dermopathy, acanthosis nigricans, lax fibroids, pruritus and xerosis are also very frequent and are considered cutaneous markers of diabetes. This review focuses on the specific and non-specific dermatological manifestations of diabetes, as well as those related to its treatment. These manifestations can be a reflection of the current or previous metabolic state of the diabetic patient and its timely identification allows guiding the primary diagnosis, to suspect a prediabetic state or to optimize the treatment of the disease in already diagnosed individuals.

\section{KEY WORDS}

Dermatology; Diabetes Mellitus; Skin Manifestations

\section{INTRODUCCIÓN}

La piel actúa como una barrera protectora frente al medio ambiente, regula la temperatura, absorbe la radiación ultravioleta (UV) \& posee capacidades propias, como órgano inmune y endocrino, en permanente interacción con los óroganos internos ${ }^{(1)}$.

Sin olvidar sus dimensiones $\left(2 \mathrm{~m}^{2}, 8-10 \mathrm{~kg}\right)$ y su condición de órogano endocrino visible, la exploración física permite identificar alteraciones que afectan directamente la piel y cambios relacionados con la disfunción de otros órganos endocrinos como la hipófisis, la tiroides, las paratiroides, el timo, las glándulas suprarrenales, el páncreas y las gónadas, entre otras $^{(1,2)}$

Según la Federación Internacional de Diabetes (IDF, siola en inglés), Colombia es el segundo país de la región con mayor número de adultos con diabetes. Se estima que hay 2.836 .500 pacientes diagnosticados y una prevalencia del $8,4 \%$, cercana a la cifra global $(9,3 \%)^{(3)}$.
Se considera que por lo menos un tercio de los diabéticos tendrán alguna manifestación dermatológica durante el curso de su enfermedad ${ }^{(4,5)}$. La frecuencia y presentación clínica de tales manifestaciones varía según la edad de los pacientes, la etnia, el tipo de diabetes $y$ el control de la enfermedad, entre otros factores. Así, en la diabetes tipo 2 la prevalencia de estos cambios cutáneos es mucho mayor. Las infecciones ocurren hasta en el $20 \%$ de todos los pacientes diagnosticados con ella, con un predominio de la micosis, que afecta los pies, las uñas y los pliegues en los diabéticos tipo $2 y$, de las verrugas virales en los tipo 1. Entre las manifestaciones no infecciosas, las más frecuentes en ambos tipos de la enfermedad son la xerosis, la dermopatía diabética y el prurito ${ }^{(6)}$.

Los cambios cutáneos pueden ser la primera manifestación de la diabetes, de un control subóptimo o de un estado prediabético ${ }^{(5)}$. Cuando estos cambios son infradiagnosticados o subestimados otros desenlaces de mayor riesgo, como el pie diabético, pueden generar complicaciones graves. Por lo tanto, su prevención, reconocimiento y tratamiento oportuno permiten reducir la morbilidad en estos pacientes.

El objetivo de esta revisión es describir las características clínicas de estas manifestaciones, su relación con la fisiopatología de la diabetes y sus principales aspectos terapéuticos.

\section{FISIOPATOLOGÍA}

Los cambios en la piel del paciente diabético ocurren como resultado de diversas alteraciones bioquímicas, estructurales y funcionales. Sin embargo, la hiperglicemia y los productos finales de glicación avanzada (AGEs, sigla en inglés) son Ios factores más importantes y los mejor estudiados ${ }^{(6)}$. La hiperglicemia aumenta la expresión del inhibidor de cinasas dependientes de ciclina (CDK, sigla en inglés), lo cual inhibe la proliferación, diferenciación y migración de los queratinocitos por interrupción del ciclo celular (7). El bloqueo de la vía de señalización de la insulina reduce la expresión de queratinas, con esto se modifica la estructura del queratinocito y se altera la función de barrera de la piel ${ }^{(7)}$. La hiperolicemia también inhibe la síntesis de proteínas, induciendo apoptosis en las células endoteliales, disminución de la síntesis 
de óxido nítrico y fallas en los procesos de fagocitosis $y$ quimiotaxis ${ }^{(6)}$.

Por otro lado, Ios AGEs favorecen la formación y acumulación de especies reactivas de oxígeno que alteran el funcionamiento de proteínas intra y extracelulares que inducen la síntesis de citocinas proinflamatorias a través de la cascada de señalización del factor nuclear $\mathrm{k} \beta$ (NF-k $\beta)^{(8)}$. La acumulación de la mayoría de estos productos ocurre en las proteínas de las membranas basales, en las proteínas circulantes y en las estructurales como la elastina y el colágeno, donde interactúan con el receptor de membrana celular específico (RAGE, sigla en inglés) que se expresa en fibroblastos, dendrocitos dérmicos y queratinocitos, entre otras células, alterando sus propiedades mecánicas con la pérdida de elasticidad, flexibilidad y solubilidad, lo que limita su funcionalidad ${ }^{(9)}$.

En cuanto a los efectos de la diabetes sobre la hidratación de la piel, los resultados de las observaciones clínicas y experimentales son controversiales. La alta prevalencia de xerosis entre los diabéticos (44\%) se ha relacionado tanto con alteraciones en la función de barrera de la piel como con las variaciones en la humedad del ambiente ${ }^{(6,10)}$. Sin embargo, aunque la xerosis no sea clínicamente evidente, el proceso natural de recambio epidérmico está alterado ${ }^{(6)}$. También se ha documentado una menor actividad de las glándulas sebáceas ${ }^{(11)}$ y un desequilibrio en la composición de los lípidos del estrato córneo, dado por la reducción en los niveles de los ácidos grasos libres ${ }^{(12)}$. Por lo tanto, las anormalidades que se presentan en la función de la barrera de la piel de los diabéticos podrían ser consecuencia de su fragilidad estructural y del desarrollo defectuoso de sus células epidérmicas.

Finalmente, la formación de AGEs también contribuye a la microvasculopatía que, en combinación con la neuropatía periférica, favorece el desarrollo del síndrome del pie diabético ${ }^{(4)}$, la complicación cutánea más grave y de peor pronóstico. Las alteraciones en la temperatura y sensibilidad, así como las deformidades anatómicas de los dedos de los pies, secundarias a la neuropatía motora, incrementan la presión plantar con la consecuente formación de úlceras que progresan debido a la disfunción que sufren los diabéticos en todas las fases del proceso de cicatrización ${ }^{(13)}$. La neuropatía autonómica genera además anhidrosis y vasodilatación, con formación de laceraciones y fisuras ${ }^{(6)}$ que son otra puerta de entrada para microorganismos potencialmente patógenos.

\section{MANIFESTACIONES CUTÁNEAS DE LA DIABETES}

Las manifestaciones cutáneas más comunes son la acantosis nioricans (50-60\%), la dermopatía diabética (30-60\%), el escleredema diabeticorum (2,5-14 \%) y las complicaciones secundarias a los cambios en la microvasculatura cutánea ${ }^{(11)}$. Las ampollas (bullosis diabeticorum) son infrecuentes (prevalencia entre 0,16 y $0,5 \%$ ), pero también se consideran un marcador cutáneo distintivo de la diabetes ${ }^{(14)}$.

En uno de los textos clásicos de dermatología se clasificaron inicialmente las manifestaciones cutáneas de la diabetes según su asociación con alteraciones metabólicas, vasculares, neurológiicas o inmunológicas, además de aquellas con patogenia desconocida ${ }^{(15)}$. Posteriormente, otros autores han incluido las infecciones cutáneas que afectan al paciente diabético y las complicaciones de la terapia antidiabética ${ }^{(15,16)}$.

A continuación, se revisan las principales manifestaciones cutáneas de la diabetes a partir de su etiología (Tabla 1).

\section{LESIONES POR MICROANGIOPATÍA DIABÉTICA}

\section{Dermopatía diabética (DD)}

Es el trastorno no infeccioso más común en los diabéticos ${ }^{(17)}$. Su incidencia se estima entre el 7 y el 70 $\%$ en mayores de 50 años con diabetes de larga data. Su causa se desconoce, pero se asocia con complicaciones microvasculares ${ }^{(18)}$. Se caracteriza por la aparición de pequeñas placas asintomáticas de color pardo, redondeadas y atróficas, de $1 \mathrm{~cm}$ de diámetro, localizadas bilateralmente en las regiones pretibiales ${ }^{(4)}$, que ocasionalmente se extienden a los muslos, el tronco e hipogastrio. La intensidad del pigmento se relaciona con el grado de atrofia. El diagnóstico se basa en la presentación clínica, aunque la biopsia de piel está indicada en casos atípicos o lesiones generalizadas. EI tratamiento es poco efectivo y las lesiones pueden persistir indefinidamente o involucionar de forma espontánea ${ }^{(5)}$. 
Tabla 1. Clasificación de las manifestaciones cutáneas de la diabetes según su etiología

\begin{tabular}{|c|c|c|c|c|}
\hline $\begin{array}{l}\text { Lesiones por } \\
\text { microangiopatía } \\
\text { diabética }\end{array}$ & $\begin{array}{l}\text { Neuropatía } \\
\text { diabética }\end{array}$ & $\begin{array}{l}\text { Infecciones } \\
\text { cutáneas }\end{array}$ & $\begin{array}{l}\text { Resistencia a } \\
\text { la insulina }\end{array}$ & $\begin{array}{c}\text { Dermatosis asociadas con la } \\
\text { diabetes mellitus }\end{array}$ \\
\hline \multirow{5}{*}{$\begin{array}{l}\text { Dermopatía } \\
\text { diabética }\end{array}$} & \multirow[t]{13}{*}{ Pie diabético } & Infecciones & Acantosis & Trastornos esclerodermiformes \\
\hline & & por hongos & nigricans & Granuloma anular \\
\hline & & & & Necrobiosis lipoídica \\
\hline & & & & Prurito \\
\hline & & & & Alopecia en las piernas \\
\hline \multirow{4}{*}{ Rubeosis faciei } & & & & Carotenodermia \\
\hline & & & & Xantomas eruptivos \\
\hline & & & & Vitiligo \\
\hline & & & & Liquen plano \\
\hline \multirow{4}{*}{$\begin{array}{l}\text { Bullosis } \\
\text { diabeticorum }\end{array}$} & & & & Colagenosis perforante reactiva \\
\hline & & Intecciones & Fibromas laxos & Psoriasis \\
\hline & & por hongos & & Reacciones a la insulina \\
\hline & & & & Reacciones por antidiabéticos orales \\
\hline
\end{tabular}

Fuente: creación propia

\section{Rubeosis faciei}

Es un eritema persistente que afecta la cara y el cuello en el $7 \%$ de los diabéticos ${ }^{(5)}$, debido a la pérdida del tono vasoconstrictor, por lo que se recomienda evaluar a estos pacientes para detectar otras microangiopatías, como la retinopatía y la nefropatía. El tratamiento de la rubeosis faciei es el control glucémico estricto, evitar el alcohol, la cafeína y otros vasodilatadores $^{(4,5)}$.

\section{Bullosis diabeticorum (ampollas del diabético)}

Ocurre entre el 0,16 y el 0,5\% de los diabéticos ${ }^{(14,19)}$. Aunque es poco común se considera un marcador distintivo de diabetes mellitus y afecta a pacientes con enfermedad de larga evolución, complicaciones microvasculares (neuropatía, nefropatía y retinopatía) y mal control metabólico. Su fisiopatología aún es desconocida, pero se acepta que la microangiopatía causa fragilidad cutánea, lo que favorece el desprendimiento subepidérmico y la formación de la ampolla (4). Clínicamente, se observan ampollas grandes, tensas, de contenidos hemorrágicos o claros, indoloras y no pruriginosas, con aparición repentina y espontánea en las regiones acrales ${ }^{(20)}$, especialmente, en las piernas y pies, a menudo son bilaterales, asimétricas y desaparecen espontáneamente sin dejar cicatrices después de 2 a 6 semanas ${ }^{(21)}$. Pese a que la sospecha clínica es importante, se le considera un diagnóstico de exclusión, pues se puede confundir con otras dermatosis ampollosas como la porfiria cutánea tarda y el penfigoide ampolloso (Figura 1). La biopsia de piel permite confirmar el diagnóstico ${ }^{(22)}$. Se recomienda protección cutánea y manejo preventivo con antibióticos tópicos, además del inmediato control metabólico ${ }^{(5,22)}$.

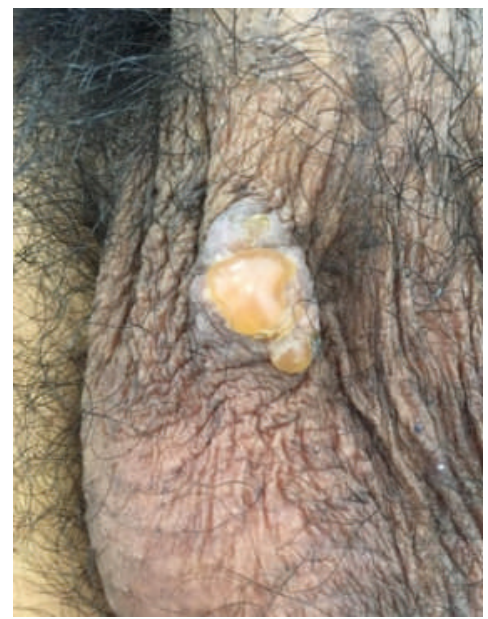

Figura 1. Ampollas tensas en el escroto de un paciente diabético tratado con sitagliptina. La biopsia de piel permitió confirmar el diagnóstico de penfigoide ampolloso. Fuente: creación propia 


\section{Neuropatía diabética}

La neuropatía diabética afecta al 50 \% de los adultos con diabetes en algún momento de su vida. Se asocia con una gran morbilidad $y$ discapacidad debido al riesgo de sufrir fracturas por caídas, úlceras y amputación de miembros inferiores ${ }^{(23)}$. Se considera un trastorno neurodegenerativo del sistema nervioso periférico cuyo blanco inicial son los axones sensitivos, aunque luego se afectan los autonómicos y, posteriormente, Ios motores. EI daño en la microvasculatura de los nervios periféricos es un factor determinante en la patogenia de la neuropatía diabética ${ }^{(24)}$. Sin embargo, la lesión de la fibra nerviosa es consecuencia de la interacción de factores vasculares, metabólicos e inmunológicos ${ }^{(25)}$.

Su presentación clínica incluye diferentes patrones sindromáticos: polineuropatía distal simétrica (PDS), neuropatía autonómica diabética (NAD), neuropatía de fibras pequeñas, radiculoplexopatías o mononeuropatías ${ }^{(26,27)}$. La PDS es la modalidad más frecuente ${ }^{(23)}$, su prevalencia es del $25 \%$ en individuos con el perfil clásico del paciente con diabetes mellitus tipo 2 de América Latina (65 años, 11 años desde el diagnóstico) ${ }^{(27)}$. La PDS inicia en áreas distales de las extremidades siguiendo un patrón en "guantes y medias" con síntomas como el adormecimiento, hormigueo, dolor lancinante y, en algunos casos, debilidad muscular. Puede preceder la aparición de lesiones cutáneas y acompañarse de hipohidrosis o anhidrosis ${ }^{(28)}$, cambios en la transpiración que generalmente afectan las extremidades inferiores, causan xerosis y fisuras en la piel, que favorece el ingreso de patógenos.

Las úlceras crónicas del pie (pie diabético) ocurren entre el 15 y el $25 \%$ de todos los diabéticos, aparecen después de un trauma debido a las alteraciones en la sensibilidad (60-70\%) o por isquemia vascular periférica ${ }^{(16)}$. Estos pacientes tienen una mortalidad alta $y$ el $50 \%$ fallece dentro de los 3 años posteriores a una amputación ${ }^{(5)}$. El tratamiento de la neuropatía diabética es básicamente preventivo y está orientado al manejo de los síntomas. El control oportuno de factores de riesgo como la hiperglucemia, la dislipidemia y la insulinorresistencia ayuda a prevenir la aparición y retardar la progresión tanto del daño neural como de las complicaciones microvasculares ${ }^{(24,26)}$.

\section{Infecciones cutáneas}

Las micosis son el grupo de infecciones cutáneas más frecuentes en los diabéticos ${ }^{(29,30)}$. La más común es la candidiasis ${ }^{(31)}$, que puede ser la primera manifestación de la enfermedad. EI manejo implica un estricto control olucémico además del tratamiento antimicótico, tópico o sistémico, según la localización y extensión de las lesiones. Otras micosis superficiales comunes en los diabéticos son la pitiriasis versicolor y las dermatofitosis ${ }^{\left({ }^{6.32}\right)}$. En los pies, la infección causada por dermatofitos ${ }^{(19,20)}$, además de afectar las uñas, se asocia con una formación de fisuras en los espacios interdigitales y las plantas, lo que favorece el riesgo de sufrir infecciones secundarias. Las infecciones bacterianas por Staphylococcus o Pseudomonas pueden ser leves o graves y presentarse como forúnculos, abscesos o celulitis. La erisipela recurrente también es frecuente entre los diabéticos. La otitis externa por Pseudomonas puede ser grave y causar mastoiditis y osteomielitis del hueso temporal, con daño en los nervios y las meninges ${ }^{(33)}$.

\section{RESISTENCIA A LA INSULINA}

\section{Acantosis nigricans}

Se caracteriza por el engrosamiento e hiperpigmentación de la piel, que adquiere un aspecto aterciopelado, principalmente en los pliegues (axilas, cuello, ingles, ombligo y codos) ${ }^{(17)}$ y en el dorso de las manos, sobre las articulaciones metacarpofalángicas ${ }^{(34)}$.

EI término acantosis nigricans (AN) se acuñó a finales del siglo XIX para hacer referencia a una manifestación dermatológica observada en pacientes con neoplasias maliognas. Hasta 1976 se reconoció su relación con la resistencia a la insulina y, desde entonces, se han propuesto diversas clasificaciones según su etiología, extensión y localización ${ }^{(35)}$. Sin embargo, el término AN describe cambios reactivos en la piel que nunca sufren una transformación neoplásica ${ }^{(36)}$, por lo tanto, en la actualidad no se utilizan los términos "AN maliogna", "AN beniogna" o "pseudoacantosis".

Cuando la AN es de origen endocrino, se presenta con mayor frecuencia en individuos de fototipo oscuro: indios, africanos, afroamericanos y mestizos latinoamericanos ${ }^{(34,35,37)}$, con una incidencia variable entre las poblaciones estudiadas. 
En los diabéticos, la AN tiene una relación directa con los niveles de insulina y la insulinorresistencia: el incremento en los niveles circulantes de la hormona favorece su unión a los receptores del factor de crecimiento similar a la insulina tipo 1 (IGF-1, sigla en inglés), con lo cual, se produce hiperplasia epidérmica ${ }^{(38)}$. Se desconocen los mecanismos responsables de la hiperpiomentación y de la localización característica en los pliegues ${ }^{(36)}$.

El control del peso y de la hiperinsulinemia constituyen las primeras etapas del tratamiento de la AN. Los queratolíticos tópicos como la urea, el lactato de amonio, ácido salicílico y algunos retinoides (tretinoína, adapaleno y tazaroteno) se han descrito como alternativas terapéuticas en lesiones localizadas, pero, generalmente, son poco efectivos ${ }^{(37)}$. Los retinoides orales como el acitretín y la isotretinoína no se recomiendan debido al riesgo de elevación de los lípidos sanguíneos. Procedimientos como la dermoabrasión y el láser abrasivo pueden mejorar el aspecto de la piel, aunque se desconocen sus efectos a largo plazo.

\section{Fibromas laxos o acrocordones}

Estos también son consecuencia de la hiperproliferación de queratinocitos que genera la hiperglucemia y el incremento en los niveles circulantes de la insulina. Las lesiones son pápulas hiperpiơmentadas o eucrómicas y asintomáticas, se localizan en los párpados, el cuello, las axilas, el escroto y las ingles. La presencia de lesiones numerosas o de gran tamaño puede ser un marcador de diabetes mellitus ${ }^{(10)}$. No es necesario ningún tratamiento, se intervienen por motivos estéticos o por molestias secundarias al roce o trauma de ellos ${ }^{(16)}$. Dentro de las técnicas descritas para su resección se incluyen el afeitado y la ablación con tijeras, la crioterapia y la electrodesecación ${ }^{(21)}$, todas con alto riesgo de recurrencia.

\section{TRASTORNOS DE LA PIEL ASOCIADOS CON LA DIABETES MELLITUS}

\section{Trastornos esclerodermiformes}

Los diabéticos pueden tener un engrosamiento y endurecimiento de la piel, esto ocurre hasta en el $50 \%$ de los casos. Sus causas podrían ser la glucosilación no enzimática del colágeno, la hidratación excesiva del mismo por grupos poliol y la mayor extravasación (por glucosilación) de la albúmina ${ }^{(39)}$. Los siguientes son algunos de ellos:

Escleredema diabeticorum: generalmente ocurre en hombres con diabetes insulinodependiente y control metabólico deficiente ${ }^{(5)}$. Su prevalencia varía entre el 2,5 y el $14 \%{ }^{(21)}$. Se produce por glicosilación de las fibras de colágeno que altera su degradación. Además, la glucosa puede estimular la proliferación de fibroblastos y la síntesis de matriz extracelular. Por otro lado, la hiperinsulinemia incrementa la producción de colágeno, dando lugar a un engrosamiento simétrico y difuso con un endurecimiento de la piel por exceso de mucopolisacáridos ácidos en la dermis ${ }^{(40)}$, afectando el cuello, el tronco y los miembros superiores, evitando las manos y los pies (Figura 2). En muchos casos, el escleredema no es fácil de identificar durante la inspección, para evidenciarlo se requiere de la palpación de la zona indurada. Entre los tratamientos descritos se incluye agentes inmunosupresores (ciclosporina y metotrexato), pentoxifilina, inmunoglobulina intravenosa, penicilamina, corticosteroides sistémicos e intralesionales, colchicina, radioterapia y fototerapia con luz ultravioleta $A$ (UVA) ${ }^{(41)}$. En general, la enfermedad es autolimitada. Sin embargo, hay casos graves con progresión que pueden causar alteraciones en el movimiento de los hombros y comprometer la función respiratoria ${ }^{(40)}$.

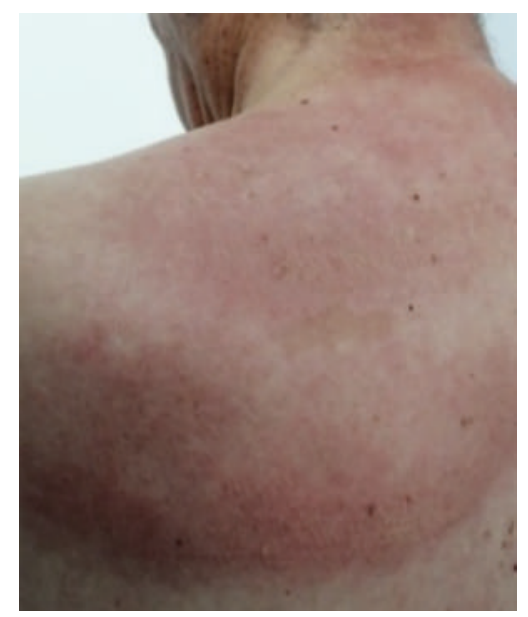

Figura 2. Escleredema diabeticorum. Paciente con diabetes tipo 2, se observa en el dorso y en la nuca el engrosamiento y el eritema difuso de la piel. Fuente: creación propia 
Escleroartropatía diabética: es una complicación temprana que afecta a los diabéticos tipo 1 y 2. Clínicamente, se observan pápulas agrupadas en el dorso de los dedos de las manos. En etapas avanzadas de induración hay contracturas flexoras responsables del "signo de la oración" en las manos, debido a la falta de contacto de la superficie palmar ${ }^{(42)}$. EI control metabólico estricto es necesario para detener su progresión, además, de la fisioterapia para mantener la movilidad. Asimismo, la infiltración de corticoides en la vaina del tendón flexor palmar puede ser útil ( ${ }^{43)}$.

Pápulas de Huntley: son múltiples micropápulas induradas que afectan las superficies extensoras y la región periungueal de los dedos de las manos. Son más comunes en la diabetes tipo 2. Aunque pueden asociarse con xerosis $y$ prurito, generalmente, son asintomáticas. En la histología se observa hiperqueratosis con engrosamiento del tejido conectivo. EI tratamiento consiste en el control glucémico. Se ha descrito involución parcial de ellas con lactato de amonio al $12 \%$, además, de corticoides tópicos e hidratantes ${ }^{(20)}$.

\section{Granuloma anular (GA)}

Es una dermatosis beniogna y autolimitada que predomina en áreas expuestas a trauma como las manos, la región pretibial $y$ las superficies extensoras de las extremidades. Presenta diferentes variantes clínicas, pero las más comunes son GA localizado, generalizado y subcutáneo ${ }^{(22)}$. Afecta principalmente a adultos jóvenes sin endocrinopatías, pero la forma generalizada se asocia con la enfermedad tiroidea, hepatitis B y C, infección por el virus deI VIH y diabetes mellitus tipo $2^{(4)}$.

Su etiología es desconocida, pero podría tratarse de una reacción de hipersensibilidad retardada tipo IV (5). Se cree que el estímulo antigénico, infeccioso o no, desencadena la liberación de linfocinas de parte de los linfocitos previamente activados, estas estimulan la síntesis y la actividad de la colagenasa produciendo una reacción inflamatoria que determina la formación de granulomas. La asociación entre GA y diabetes aún es controvertida ${ }^{(16,18)}$. Sin embarogo, hasta el $77 \%$ de los pacientes con la variante generalizada son diabéticos ${ }^{(44)}$, y estos tienen un mayor riesgo de recurrencia de la enfermedad, en comparación con los individuos no diabéticos ${ }^{(5)}$.
Las lesiones comienzan como pápulas eucrómicas firmes, que luego toman una configuración anular con leve hiperpiomentación central. La mayoría de estas desaparecen espontáneamente sin dejar cicatrices. Cuando la presentación es atípica o, existe alguna duda en el diagnóstico, se recomienda su confirmación por medio de biopsia de piel.

Los corticoides intralesionales o tópicos de alta potencia y los inhibidores de la calcineurina son efectivos en la variante localizada. Para la generalizada se ha descrito fototerapia UVA con psoralenos (PUVA, sigla en inglés), tratamiento sistémico con antimaláricos, dapsona o metotrexato y terapia biológica con anti-TNF ${ }^{(4,22)}$.

\section{Necrobiosis lipoídica (NL)}

Es una enfermedad granulomatosa crónica caracterizada por la degeneración del colágeno, el engrosamiento de la pared endotelial y la aparición de depósitos de grasa ${ }^{(18)}$. La causa primaria de la degeneración parece ser una vasculitis autoinmune ${ }^{(20)}$. Es una dermatosis infrecuente entre la población general, dos tercios de los casos ocurren en pacientes diabéticos ${ }^{(4)}$. Las lesiones se presentan después del diagnóstico de la diabetes en el $62 \%$ de los casos y su incidencia entre los diabéticos varía del 0,3 al 1,2\% ${ }^{(45)}$. También se asocia con una disfunción tiroidea, la artritis reumatoide y enfermedad de Crohn, puede confundirse con otras dermatosis como el granuloma anular, mixedema pretibial, eritema nodoso, xantogranuloma necrobiótico y sarcoidosis cutánea, entre otras ${ }^{(46)}$.

Las lesiones afectan la piel en áreas pretibiales, muslos, región poplítea y pies, con distribución bilateral y simétrica. Inician con pápulas eritematosas que evolucionan a placas pardo-amarillas con un centro atrófico y telangiectasias ${ }^{(5)}$. Su aspecto amarillento en el área central de la placa se debe al adelgazamiento de la dermis, que hace más visible la grasa subcutánea $(21,47)$. EI $35 \%$ de las lesiones se ulceran produciendo dolor; ocurre una resolución espontánea entre el 10 y el $20 \%$ de los casos ${ }^{(5)}$. También se ha descrito su aparición sobre cicatrices, a veces de vacunas, y en lesiones antiguas de esclerodermia, también una transformación maliogna del epitelio escamoso (carcinoma escamocelular) en casos de ulceración crónica ${ }^{(45)}$. EI diagnóstico se confirma por medio de la histopatología, donde se observa la necrobiosis (necrosis de las fibras de colágeno). 
Además del control metabólico y la prevención del trauma para reducir el riesgo de ulceración e infección, el tratamiento es similar al del granuloma anular, con corticoides intralesionales o tópicos de alta potencia, inhibidores de la calcineurina y PUVA ${ }^{(22)}$. Se ha descrito el manejo con pentoxifilina, ácido acetil salicílico, colchicina, antimaláricos, láser de colorante pulsado y terapia biológica ${ }^{(5)}$.

\section{Prurito}

El prurito es la sensación que conduce al deseo de rascarse. Se estima que puede afectar entre del 12,5 y el $25 \%$ de los diabéticos ${ }^{(4,48,49)}$, aunque es probable que una directa asociación con la xerosis determine su aparición recurrente en un mayor número de pacientes. A pesar de que no existe una relación entre la intensidad del prurito y la gravedad de la enfermedad, el control de la glicemia ayuda a aliviar el síntoma cuando el prurito es generalizado ${ }^{(50,51)}$.

En cuanto a su fisiopatología, la disfunción de los nervios simpáticos altera la termorregulación y causa hipohidrosis y xerosis ${ }^{(4)}$, además de la alteración del umbral sensitivo de las fibras $C$ subepidérmicas secundaria a la neuropatía ${ }^{(48)}$. Asimismo, el prurito de predominio truncal se considera una manifestación de neuropatía en los pacientes diabéticos ${ }^{(48)}$, y el prurito del cuero cabelludo puede ser una de las primeras manifestaciones de la enfermedad en la población geriátrica ${ }^{(52)}$.

Otros autores consideran que el prurito en los diabéticos puede ser una reacción adversa al tratamiento farmacológico y, que solo el prurito genital y anal, es más frecuente en los diabéticos que en la población general, probablemente, debido al mayor riesgo de infección por Candida ${ }^{(5,51,53)}$.

En todos los casos se recomienda el control de la xerosis con emolientes y cuidados generales como evitar el rascado y el trauma en las extremidades y en las zonas afectadas por cualquier dermatosis, utilizar material desechable para manicura y pedicura, corte recto de las uñas, aseo sin fricción de la piel evitando el uso de jabones alcalinos y antibacteriales, evitar el contacto con textiles irritantes como la lana y las fibras sintéticas (nylon y poliéster), evitar el uso de suavizantes y detergentes perfumados para el lavado de las prendas de vestir, enjuagar bien para remover los residuos de jabón, uso de chanclas para duchas y áreas húmedas, secado de pliegues $y$ espacios interdigitales con toallas limpias, evitar la exposición prolongada al calor $y$ al agua caliente, duchas cortas con agua tibia o fría, inspección frecuente de las extremidades con el objetivo de identificar heridas o infecciones y el uso de calzado amplio y cómodo. Cuando el prurito es localizado o se acompaña de lesiones nodulares secundarias al rascado crónico, se pueden utilizar corticoides tópicos o intralesionales y capsaicina. Para el manejo del prurito generalizado se ha descrito el tratamiento con antihistamínicos no sedantes, talidomida e inhibidores de la recaptación de serotonina ${ }^{(16.52)}$. La fototerapia con luz ultravioleta $B$ de banda estrecha se reserva para casos refractarios.

\section{Alopecia en las piernas}

Se produce por el daño en la microcirculación y retraso en la regeneración celular, cambios propios de la diabetes, que afectan el ciclo de crecimiento del folículo piloso. Se puede considerar como un marcador cutáneo de la diabetes y se encuentra hasta en el 6 \% de los casos ${ }^{(54)}$.

\section{Carotenodermia}

La piel y las uñas amarillas se han atribuido a la carotenodermia en los pacientes con diabetes ${ }^{(21)}$. Su fisiopatología, probablemente, implica una glicación no enzimática por mal control glucémico que promueve el aumento en los niveles séricos de carotenoides, con el consiguiente depósito en la piel ${ }^{(16)}$. No hay tratamiento para este fenómeno.

\section{Xantomas eruptivos}

Estas lesiones se observan especialmente cuando hay elevación de los trioglicéridos (> $700 \mathrm{mg} / \mathrm{dL}$ ) como consecuencia del mal control metabólico. Las lesiones pueden aparecer como pápulas cupuliformes amarillas de 1-2 mm con una base eritematosa y suelen ubicarse en las superficies extensoras de las extremidades y las caderas ${ }^{(18)}$. Los xantomas eruptivos tienden a resolver luego de controlar el metabolismo de los carbohidratos y lípidos ${ }^{(21)}$.

\section{Vitiligo}

Es una enfermedad crónica autoinmune que puede estar asociada con Ia DM tipo $1^{(17,33)} y$ afectar entre el 1 y el $7 \%$ de todos los pacientes diabéticos, en contraste 
con el 0,2-1 \% de la población general. Su causa es multifactorial: una combinación de factores autoinmunes, genéticos, neurológicos y humorales que producen sustancias que llevan a la destrucción de los melanocitos. Factores ambientales como la infección o el traumatismo (fenómeno de Koebner) también pueden contribuir a la aparición de las lesiones. Se manifiesta como máculas acrómicas ubicadas alrededor de los orificios naturales, en las regiones extensoras, en el tórax y en el abdomen ${ }^{(5)}$. En las lesiones pequeñas y localizadas el tratamiento de elección son los corticoides tópicos y, en el vitiligo generalizado el tratamiento más efectivo es la fototerapia con luz ultravioleta B (UVB) de banda estrecha ${ }^{(33)}$. Los inhibidores tópicos de la calcineurina como el tacrolimus tienen menos efectos adversos en comparación con los corticosteroides tópicos ${ }^{(16)}$. Los tratamientos cosméticos como el maquillaje de camuflaje y micropigmentación son una buena opción que permite mejorar la calidad de vida de estos pacientes.

\section{Liquen plano}

Alrededor de una cuarta parte de los pacientes con liquen plano tiene diabetes ${ }^{(16)}$. Clínicamente, se observan pápulas eritematosas poligonales pruriginosas con estrías blancas que, inicialmente, afectan los tobillos o las muñecas y después se generalizan formando placas que se pigmentan. Para el tratamiento se utilizan corticoides tópicos, inhibidores tópicos de la calcineurina, fototerapia, retinoides sistémicos, corticosteroides sistémicos, metotrexato, hidroxicloroquina y dapsona ${ }^{(21)}$.

\section{Colagenosis perforante reactiva adquirida (CPRA)}

Es una enfermedad caracterizada por la eliminación transepidérmica del tejido conectivo en la dermis ${ }^{(18)}$. Se observa en diabéticos y pacientes con insuficiencia renal crónica e hiperuricemia ${ }^{\left({ }^{16}\right)}$. Su patogenia se desconoce y para el diagnóstico es fundamental la biopsia de piel, donde se observa la eliminación transepitelial del colágeno en la dermis papilar. Clínicamente, se presenta con nódulos umbilicados con un tapón hiperqueratósico o placas eritematosas pruriơinosas de 2-10 mm con costras adherentes ubicadas en las extremidades, el tronco y el dorso de las manos ${ }^{(21,41)}$. Las lesiones pueden involucionar después de meses si se evitan los traumas y el rascado. Como alternativas de tratamiento se han descrito corticoides tópicos, intralesionales y sistémicos, retinoides orales y alopurinol ${ }^{(5,41)}$

\section{Psoriasis}

Es una enfermedad inflamatoria crónica con una variable afectación de la piel: desde unas pocas placas hasta extensas áreas con numerosas lesiones. Cuando la extensión es mayor al 10 \% de la superficie corporal puede asociarse con artritis psoriásica y síndrome metabólico. Se cree que los pacientes con psoriasis severa tienen un rieso dos veces mayor de desarrollar diabetes, en comparación con la población gene$\mathrm{raI}^{(18)}$. El diagnóstico de psoriasis suele ser clínico y el tratamiento depende del tipo y la extensión de las lesiones. Se pueden utilizar corticoides tópicos en casos leves e, inmunosupresores y terapia biológica en los severos que deben complementarse con un estilo de vida saludable, medidas para controlar el estrés y eđucación nutricional.

\section{Reacciones causadas por la insulina}

La aplicación continua de insulina puede provocar lipohipertrofia, lipoatrofia, infecciones locales o alergia al medicamento. La lipohipertrofia es la reacción local más frecuente afectando al $27 \%$ de los diabéticos. Los hallazoos clínicos son nódulos cutáneos de tamaño variable. Esta complicación también afecta la absorción local de la insulina y resuelve espontáneamente al cambiar el sitio de aplicación ${ }^{(47)}$. La lipoatrofia se caracteriza por la atrofia del tejido adiposo en el sitio de la inyección de insulina. El riesgo de infecciones bacterianas locales depende del número de inyecciones diarias, siendo menos comunes en pacientes que usan bombas de insulina. La alergia a la insulina puede variar desde síntomas localizados como el eritema, las pápulas, vesículas y el prurito, hasta una reacción generalizada con urticaria $y$ angioedema que amenazan la vida. El uso de insulina altamente purificada $y$ recombinante reduce la prevalencia de reacciones a la insulina ${ }^{(16)}$

\section{Reacciones cutáneas inducidas por antidiabéticos orales}

Los antidiabéticos orales pueden causar reacciones como el eritema multiforme, vasculitis leucocitoclástica, erupciones por medicamentos $y$ fotosensibilidad, 
entre otras. Los derivados de las sulfonilureas como la ğlibenclamida son los fármacos más asociados con las reacciones en la piel: fotosensibilidad, erupciones liquenoides o psoriasiformes e incluso pénfigo vulgar (16). La metformina es una biouanida que actúa en la supresión de la gluconeogénesis hepática; se ha asociado con casos de vasculitis leucocitoclástica y erupción psoriasiforme. La acarbosa actúa inhibiendo la enzima a-glucosidasa, se ha descrito como posible desencadenante del eritema multiforme y pustulosis exantemática generalizada. Asimismo, estudios recientes han sugerido que el uso de inhibidores de dipeptidil peptidasa 4 (iDPP-4) se asocia con un riesgo mayor de desarrollar penfigoide ampolloso ${ }^{(55,56)}$.

\section{CONCLUSIONES}

La evaluación clínica de la piel, como órgano inmune $y$ endocrino, permite reconocer las manifestaciones cutáneas de muchas enfermedades sistémicas, lo cual permite orientar su diagnóstico y el tratamiento oportuno, como la diabetes mellitus, que es una de las endocrinopatías más frecuentes en Colombia con gran variedad de manifestaciones cutáneas, algunas de ellas, como la dermopatía diabética y la acantosis nigricans, se comportan como marcadores precoces de la enfermedad, de allí la importancia de su oportuno reconocimiento. Manifestaciones cutáneas de la diabetes como la bullosis diabeticorum, los trastornos esclerodermiformes y la CPRA son de difícil diagnóstico clínico, por ello, la biopsia de piel es necesaria para confirmarlos. El éxito del tratamiento de las manifestaciones cutáneas de la enfermedad depende tanto del control metabólico como del autocuidado de cada paciente.

\section{CONFLICTOS DE INTERESES}

Ninguno por declarar.

\section{REFERENCIAS BIBLIOGRÁFICAS}

1. Olaya PA, Gamboa M, Valbuena MC. Sistema inmune cutáneo y su relación con el sistema neuroendocrino. En: Tamayo MM, editor. Dermatología Clínica: Conceptos Básicos. Bogotá D.C: Panamericana; 2015. p. 23-32.
2. Zouboulis CC, Stratakis CA, Chrousos GP, Koch CA. Metabolism and skin diseases. Rev Endocr Metab Disord. 2016;17:241-6. DOI 10.1007/s11154-016-9396-6.

3. International Diabetes Federation [internet]. Brussels: IDF; 2019. [Access 2019 July 3]. Available at: http://www.diabetesatlas.org

4. Behm B, SchremI S, Landthaler M, Babilas P. Skin signs in diabetes mellitus. JEADV. 2012;26:1203-11. DOI 10.1111/j.1468-3083.2012.04475.x.

5. Murphy-Chutorian B, Han G, Cohen SR. Dermatologic manifestations of diabetes mellitus: a review. Endocrinol Metab Clin N Am. 2013;42:869-98. DOI 10.1016/j.ec1.2013.07.004.

6. De Macedo GM, Nunes S, Barreto T. Skin disorders in diabetes mellitus: an epidemiology and physiopathology review. Diabetol Metab Syndr. 2016;8:63. DOI 10.1186/s13098-016-0176-y.

7. Aoki M, Murase T. Obesity-associated insulin resistance adversely affects skin function. PLoS One. 2019;14(10):e0223528. DOI 10.1371/journal. pone. 0223528 .

8. Gkogkolou P, Böhm M. Advanced glycation end products: Key players in skin aging? Dermatoendocrinol. 2012;4:259-70. DOI 10.4161/derm.22028.

9. Cohen EN. La glicosilación no enzimática: una vía común en la diabetes y el enrejecimiento. Med Cutan Iber Lat Am. 2011;39:243-6.

10. Goyal A, Raina S, Kaushal SS, Mahajan V, Sharma NL. Pattern of cutaneous manifestations in diabetes mellitus. Indian J Dermatol. 2010;55:39-41. DOI 10.4103/0019-5154.60349.

11. Sakai S, Kikuchi K, Satoh J, Tagami H, Inoue S. Functional properties of the stratum corneum in patients with diabetes mellitus: similarities to senile xerosis. Br J Dermatol. 2005;153:319-23. DOI 10.1111/j.1365-2133.2005.06756.x.

12. Wohlrab J, Gabel A, Wolfram M, Grosse I, Neubert RHH, Steinbach SC. Age- and Diabetes-related changes in the free fatty acid composition of the human stratum corneum. Skin Pharmacol Physiol. 2018;31:283-91. DOI 10.1159/000490800.

13. Blakytny R, Jude EB. Altered molecular mechanisms of diabetic foot ulcers. Int J Low Extrem Wounds. 2009;(8)2:95-104. DOI 10.1177/1534734609337151.

14. Bustan RS, Wasim D, Yderstræde KB, Bygum A. Specific skin signs as a cutaneous marker of diabetes 
mellitus and the prediabetic state - a systematic review. Dan Med J. 2017;64:A5316.

15. Fitzpatrick's. Dermatology in General Medicine. 8. ed. New York, McGraw Hill, 2012.

16. Lima A, Illing T, Schliemann S, Elsner P. Cutaneous manifestations of diabetes mellitus: A review. Am J Clin Dermatol. 2017;18:541-53. DOI 10.1007/s40257017-0275-z.

17. Kafaie P, Shojaoddiny-Ardekani A. Skin manifestations of diabetes mellitus. IJDO. 2012;4:91-8.

18. Karadag A, OzIu E, Lavery M. Cutaneous manifestations of diabetes mellitus and the metabolic syndrome. Clin Dermatol. 2018;36:89-93. DOI 10.1016/j.clindermatol.2017.09.015.

19. Vangipuram R, Hinojosa T, Lewis DJ, Downing C, Hixson C, Salas JC, et al. Bullosis diabeticorum: A neolected bullous dermatosis. Skinmed. 2018;16:77-9.

20. Baloch GH, Memon NM, Devrajani BR, Iqbal P, Thebo NK. Cutaneous manifestations of type II diabetes meIlitus. J Liaquat Uni Med Health Sci. 2008;7:67-70.

21. Van Hattem S, Bootsma A, Thio B. Skin manifestations of diabetes. Cleve Clin J Med. 2008;75:772-4. DOI 10.3949/ccjm.75.11.772.

22. Calderón DC, Rivera A, Medina A. Diabetes mellitus y sus diferentes manifestaciones dermatológicas. Revisión de la literatura. Revista Colombiana de Endocrinología, Diabetes y Metabolismo. 2017;4(3):33-40.

23. Iqbal Z, Azmi S, Yadav R, Ferdousi M, Kumar M, Cuthbertson DJ, et al. Diabetic peripheral neuropathy: Epidemiology, diagnosis, and pha rmacotherapy. Clin Ther. 2018;40(6):828-49. DOI 10.1016/j.clinthera.2018.04.001.

24. DogiparthiSN, MuralidharK, SeshadriKG, RangarajanS. Cutaneous manifestations of diabetic peripheral neuropathy. Dermatoendocrinol. 2017;9(1):e1395537. DOI 10.1080/19381980.2017.1395537.

25. Kles KA, Vinik AI. Pathophysiology and treatment of diabetic peripheral neuropathy: the case for diabetic neurovascular function as an essential component. Curr Diabetes Rev. 2006;2(2):131-45. DOI 10.2174/157339906776818569.

26. Peltier A, Goutman SA, Callaghan BC. Painful diabetic neuropathy. BMJ. 2014;348:g1799. DOI 10.1136/ bmj.g1799.

27. Vizcaíno E, Díaz O, Hernández JD, Rodríguez M, Franco H, Gómez Y, et al. Polineuropatía diabética:
Prevalencia y factores asociados en una población colombiana. Rev ALAD. 2012;2:264-73.

28. Yasmeen B, Gupta V. Kudyar R. Cutaneous manifestations of diabetes mellitus. Int J Diab Dev Ctries. 2006;26(4):152-7. DOI 10.4103/0973-3930.33180.

29. Barrera F, Santacruz G, Zambrano M, Serrano M, Gordillo F, Palacios S. Manifestaciones cutáneas en pacientes con diabetes mellitus en un centro de atención primaria en Ecuador. Dermatol Rev Mex. 2017;61(6):457-73.

30. Kataria U, Chhillar D, Kumar H, Chhikara P. Cutaneous manifestations of diabetes mellitus in controlled and uncontrolled state. IAIM. 2015;2(2):90-3.

31. Gupta SK, Parminder-Singh M. Pattern of dermatological diseases in patients of diabetes mellitus. JPAD. 2016;26(3):214-8

32. Lugo-Somolinos A, Sanchez JL. Prevalence of dermatophytosis in patients with diabetes. J Am Acad Dermatol. 1992;26(3):408-10. DOI 10.1016/01909622(92)70063-L.

33. Mendes A, Miot H, Haddad $\nabla$. Diabetes mellitus and the skin. An Bras Dermatol. 2017;92(1):8-20. DOI 10.1590/abd1806-4841.20175514.

34. López-Alvarenga JC, Garcia-Hidalogo L, Landa-Anell MV, Santos-Gomez R, Gonzalez-Barranco J, Comuzzie A. Influence of skin color on the diagnostic utility of clinical acanthosis nigricans to predict insulin resistance in obese patients. Arch Med Res. 2006;37(6):7448. DOI 10.1016/j.arcmed.2005.12.007.

35. Karadag AS, You Y, Danarti R, AI-Khuzaei S, Chen W. Acanthosis nigricans and the metabolic syndrome. Clin Dermatol. 2018;36:48-53. DOI 10.1016/j.clindermatol.2017.09.008.

36. De Schepper S, Naeyaert JM. Acantosis nioricans. EMC - Dermatología. 2006;40(2):1-7. DOI 10.1016/ s1761-2896(06)46450-5.

37. Bahadursingh S, Mungalsingh C, Seemungal T, Teelucksingh $\mathrm{S}$. Acanthosis nigricans in type 2 diabetes: prevalence, correlates and potential as a simple clinical screening tool -a cross-sectional study in the Caribbean. Diabetol Metab Syndr. 2014;6(77). DOI 10.1186/1758-5996-6-77.

38. Edmondson SR, Thumiger SP, Kaur P, Loh B, Koelmeyer R, Li A, et al. Insulin-like growth factor binding protein-3 (IGFBP-3) localizes to and modulates proliferative epidermal keratinocytes in vivo. Br J 
Dermatol. 2005;152(2):225-30. DOI 10.1111/j.13652133.2004.06350.x.

39. Zaballos D, Garrido AM, Blasco P, Lafuente E, Pinós PJ. Manifestaciones cutáneas de la diabetes. Med Integr. 2001:38:36-42.

40. Pereira-Garzón M, Morales-Cardona CA. Escleredema de Buschke en un hombre diabético. Bol Dermatol. 2017;15:14-18.

41. Levy L, Zeichner J. Dermatologic manifestation of diabetes. J Diabetes. 2012;4:68-76. DOI 10.1111/j.17530407.2011.00151.x.

42. Cherqaoui R, McKenzie S, Nunlee-Bland G. Diabetic cheiroarthropathy: a case report and review of theliterature. Case Rep Endocrinol. 2013;257028. DOI 10.1155/2013/257028.

43. Ferreli C, Gasparini G, Parodi A, Cozzani E, Rongioletti F, Atzory L. Cutaneous manifestations of scleroderma and scleroderma-like disorders: a comprehensive review. Clin Rev Allergy Immunol. 2017:53(3):306-36. DOI 10.1007/s12016-017-8625-4.

44. Dabski K, WinkeImann RK. Generalized granuloma annulare: clinical and laboratory findings in 100 patients. J Am Acad Dermatol. 1989;20(1):39-47. DOI 10.1016/s0190-9622(89)70005-0.

45. Reid SD, Ladizinski B, Lee K, Baibergenova A, Alavi A. Update on necrobiosis lipoidica: a review of etiology, diagnosis, and treatment options. J Am Acad Dermatol. 2013;69:783-91. DOI 10.1016/j.jaad.2013.05.034.

46. Sibbald C, Reid SD, Alavi A. Necrobiosis Lipoidica. Dermatol Clin. 2015;33(3):343-60. DOI 10.1016/j. det.2015.03.003.

47. Ahmed I, Goldstein B. Diabetes mellitus. Clin Dermatol. 2006;24(4):237-46. DOI 10.1016/j.clindermatol.2006.04.009.

48. Yamaoka H, SasaKi H, Yamasaki H, Ogawa K, Ohta T, Furuta H, et al. Truncal pruritus of unknown origin may be a symptom of diabetic polyneuropathy. Diabetes Care. 2010;33(1):150-5. DOI 10.2337/dc09-0632.

49. Alizadeh N, Mirpour SH, Golmohamadi R, Darjani A, Eftekhari H, Rafiei R, et al. Chronic generalized pruritus without primary skin lesions: a longitudinal prospective observational study. Int J Dermatol. 2019;58(3):273-8. DOI 10.1111/ijd.14125.

50. Ko MJ, Chiu HC, Jee SH, Hu FC, Tseng CH. Postprandial blood glucose is associated with generalized pruritus in patients with type 2 diabetes. Eur J Dermatol. 2013;23(5):688-93. DOI 10.1684/ejd.2013.2100.

51. Polat M, Oztas P, Ilhan MN, Yalçin B, Alli N. Generalized pruritus: A Prospective Study Concerning Etiology. Am J Clin Dermatol. 2008;9(1):39-44. DOI 10.2165/00128071-200809010-00004.

52. Shevchenko A, Valdes-Rodriguez R, Yosipovitch G. Causes, pathophysiology, and treatment of pruritus in the mature patient. Clin Dermatol. 2018;36:14051. DOI 10.1016/j.clindermatol.2017.10.005.

53. Senet P, Chosidow O. Manifestaciones cutáneo-mucosas de la diabetes. EMC Dermatología. 2012;46:18. DOI 10.1016/s1761-2896(12)60831-0.

54. Timshina DK, Thappa DM, Agrawal A. A clinical study of dermatoses in diabetes to establish its markers. Indian J Dermatol. 2012;57(11):20-5. DOI 10.4103/00195154.92671.

55. Damas-Fuentes M, Díaz-Perdigones C, HernándezGarcía C, Mancha-Doblas I, Tinahones-Madueño F. Asociación de penfigoide ampolloso con inhibidores de dipeptidil peptidasa 4 en nuestro medio. Endocrinol Diabetes Nutr. 2019;66(Espec Cong 1):76.

56. Douros A, Rouette J, Yin H, Yu OHY, Filion KB, Azoulay L. Dipeptidyl Peptidase 4 Inhibitors and the risk of bullous pemphigoid among patients with type 2 diabetes. Diabetes Care. 2019;42(8):1496-503. DOI 10.2337/dc19-0409. 